\title{
Upgrading the MHD Equation of State to Include Relativistic Electrons
}

\author{
Zhigang Gong, Werner Däppen \\ Department of Physics and Astronomy, University of Southern \\ California, Los Angeles, CA90089, U.S.A.
}

\begin{abstract}
So far, the effect of relativistic electrons has not been included in the Mihalas-Däppen-Hummer (Hummer \& Mihalas 1988; Mihalas, Däppen, \& Hummer 1988; Däppen et al. 1988; hereinafter MHD) equation of state, although degeneracy was taken into account. Following the findings about the detectability of the relativistic effect in helioseismological data of the solar center (Elliot \& Kosovichev 1998; hereinafter EK98), we have upgraded the MHD equation of state to include relativistic degenerate electrons. Our numerical calculation confirms the result of EK98.
\end{abstract}

\section{Introduction}

The relativistic effect is significant when temperature is high enough that the kinetic energy of particles is non-negligible compared with their rest-mass energy. In the center of the sun, the temperature is less than $2 \times 10^{7} \mathrm{~K}$. Even at such temperatures, the relativistic effect for electrons is of course weak, with the kinetic energy being less than $0.3 \%$ of the rest-mass energy. For heavier particles, the effect is completely negligible.

However, helioseismological observations are now so precise that even such a tiny difference can be detected (EK98). Hence we have included the relativistic effect into the MHD equation of state. We compare our relativistic treatment with the approximate relativistic treatment of EK98 in a solar model.

\section{Relativistic Equations of State}

The general expressions of density, pressure and internal energy for partially degenerated electrons are given by Cox \& Giuli (1968)

$$
\begin{aligned}
& n_{e}=\frac{8 \pi \sqrt{2}}{h^{3}} m^{3} c^{3} \beta^{\frac{3}{2}}\left[F_{\frac{1}{2}}(\eta, \beta)+\beta F_{\frac{3}{2}}(\eta, \beta)\right] \\
& p_{e}=\frac{16 \pi \sqrt{2}}{3 h^{3}} m^{4} c^{5} \beta^{\frac{5}{2}}\left[F_{\frac{3}{2}}(\eta, \beta)+\frac{\beta}{2} F_{\frac{5}{2}}(\eta, \beta)\right] \\
& u_{e}=\frac{8 \pi \sqrt{2}}{h^{3}} m^{4} c^{5} \beta^{\frac{5}{2}}\left[F_{\frac{3}{2}}(\eta, \beta)+\beta F_{\frac{5}{2}}(\eta, \beta)\right]
\end{aligned}
$$


where $F_{k}(\eta, \beta)=\int_{0}^{\infty} x^{k} \sqrt{1+(\beta x / 2)} /\left(e^{x-\eta}+1\right) \mathrm{d} x$ is the generalized Fermi-Dirac integral. From this we can get the free energy and use it in the free-energy-minimization procedure of the MHD equation of state.

The generalized Fermi-Dirac integrals are very complicated and analytical expressions are only available for certain limiting cases. For our calculation, a broader range of physical conditions have to be considered. We have adopted the numerical method developed by Aparicio (1998), in which the generalized Fermi-Dirac integrals are divided into four parts, and Gauss-Legendre and Gauss-Laguerre quadrature are used to evaluate the integral in each piece. By doing this, the approximation of the generalized Fermi-Dirac integral can become essentially as good as machine precision.

\section{Results}

From our results it is shown that the relativistic correction in the Sun is very small. Its influence on the thermodynamic functions is slightly larger. EK98 quite convincingly demonstrated that this correction is the source of the difference between the adiabatic exponent $\left(\gamma_{1}=\partial \ln p / \partial \ln \rho\right)_{s}(s$ being specific entropy) of a solar model and that obtained from inversion of helioseismic data.

We compare the change of adiabatic exponent $\gamma_{\mathbf{1}}$ between relativistic and nonrelativistic treatment in our calculation and the approximation given in EK98 [their equation (22)]. Our numerical result shows a somewhat bigger difference between the relativistic calculation and the nonrelativistic approximation, although our result is similar to Fig. 2 of EK98. Detailed results will be published elsewhere.

With the relativistic effect included, the MHD equation of state can now be extended to very high-temperature plasmas, such as found in massive stars. The same relativistic upgrade becomes immediately applicable to Q-MHD, which is the recent improvement of the MHD equation of state (Nayfonov et al. 1999). Appropriate equation of state tables (described in Däppen \& Guzik 1999) will be made available.

Acknowledgments. This work was supported by the grant AST-9618549 of the NSF and the SOHO Guest Investigator grant NAG5-7352 of NASA.

\section{References}

Aparicio, J. M. 1998, ApJS, 177, 627

Cox, J. P. \& Giuli, R. T. 1968, Principles of Stellar Structure (New York: Gordon \& Breach)

Däppen, W. \& Guzik, J. A. 1999, in Variable Stars as Essential Astrophysical Tools, ed. C. Ibanoglu, NATO-ASI, (Dordrecht: Kluwer), in press

Däppen, W., Mihalas, D., Hummer, D. G., \& Mihalas, B. W. 1988, ApJ, 332, 261

Elliot, J. R. \& Kosovichev, A. G. 1998, ApJ, 500, L119

Hummer, D. G. \& Mihalas, D. 1988, ApJ, 331, 794

Mihalas, D., Däppen, W., \& Hummer, D. G. 1988, ApJ, 331, 815

Nayfonov, A., Däppen, W., Hummer, D. G., \& Mihalas, D. 1999, ApJ, in press 